\title{
DIFFERENTIATING DSM-IV ALCOHOL DEPENDENCE AND ABUSE BY COURSE: Community Heavy Drinkers
}

\author{
DEBORAH S. HASIN* \\ RONAN VAN ROSSEM \\ STEVEN MCCLOUD \\ JEAN ENDICOTT \\ Columbia University/NYS Psychiatric Institute
}

\begin{abstract}
The purpose of this study was to investigate the differences in shortterm course between DSM-IV alcohol abuse and alcohol dependence in a community sample of men and women. Eight hundred seventy-six individuals were given a baseline interview that included DSM-IV criteria for alcohol use disorders, and were followed up with a similar interview approximately one year later. Group differences were compared with chi-square tests. Multinomial logistic regression analyses were used to test group differences controlling for potential confounders. The course of alcohol abuse and dependence differed significantly from each other, and from individuals with no alcohol diagnosis at baseline. Chronicity was the most common outcome for dependence but not for abuse. Most subjects with baseline diagnoses of abuse received the diagnosis with only one symptom, repeated drunk driving. While the validity of the alcohol dependence diagnosis was supported in this community sample, the results concerning alcohol abuse remain equivocal.
\end{abstract}

The definitions of alcohol dependence in DSM-III-R, ICD-10 and DSM-IV have been based on a common underlying concept, the Dependence Syndrome (Edwards \& Gross,

*Direct all correspondence to: Deborah Hasin, Columbia University/NYS Psychiatric Institute, Box 123, 722 West 168th Street, New York, NY 10032.

JOURNAL OF SUBSTANCE ABUSE, Volume 9, pages 127-135.

Copyright $\odot 1997$ by Ablex Publishing Corporation

All rights of reproduction in any form reserved.

ISSN: 0899-3289 
1976; World Health Organization, 1981), in which "impaired control" over drinking is the main feature (World Health Organization, 1977). However, no such concept underlies alcohol abuse other than the fact that abuse differs from dependence, is diagnosed only in the absence of dependence, and is characterized by external behaviors related to drinking or drug use such as work irresponsibility, drunk driving or fights. Numerous questions have been raised about the abuse category, including whether it is a valid diagnostic category at all, whether abuse is merely a prodromal state to dependence, and what the definitions of abuse, if any, should be. An early decision to drop abuse from DSM-III-R (Rounsaville et al., 1986) was reversed after objections were made, but the DSM-III-R abuse criteria partially overlapped with the criteria for dependence. In the transition from DSM-III-R to DSM-IV, the definition of alcohol abuse was separated more clearly from dependence. Four criteria were given, an expanded number from DSM-III-R (which had only two criteria for abuse). The DSM-IV criteria for abuse included hazardous use (regardless of whether a DWI citation was received), legal problems, failure to fulfill major role obligations (formerly a DSM-III-R dependence criterion) and continued use despite social or interpersonal problems. However, the validity of the newly defined DSM-IV categories and their distinctions from each other have not been studied in terms of their course over time.

As stated by Robins and Guze many years ago (1970), follow-up study constitutes an important method of understanding the validity of disorders and their differentiation from one another. Prospective data avoid problems of recall and other distortions. However, little prospective naturalistic information has been available on alcohol abuse and dependence in untreated samples. We previously conducted secondary analyses on differences in the course of DSM-III-R abuse and dependence (Hasin et al., 1990). The data came from a small national sample of male drinkers initially interviewed in 1969 (Cahalan \& Room, 1974) and followed up four years later (Fillmore, 1987). Diagnostic concepts from the 1980's and 1990's were obviously not specifically operationalized in this older study. However, the data set contained enough information to construct approximations to DSM-III-R abuse and dependence diagnoses. In this study, the "abuse" diagnosis was represented fully. However, the construction of the "dependence" diagnoses was much more problematic, since only six of the nine DSM-III-R dependence criteria were available in the data set and tolerance had to be inferred from a quantity/frequency indicator. Results (including a subsequent re-analysis and clarification; Hasin et al., 1991a, 1991b) indicated that the four-year outcomes of those initially classified in the "abuse" and "dependence" categories differed significantly. In this study, the operationalization of the diagnostic criteria left much to be desired, the sample included only men, and the data were collected many years ago. Given the absence of other comparable information, the 1990 study began to fill a gap in knowledge. However, we concluded that future investigation should correct the problems with the early study (particularly the representation of the diagnostic criteria) and that much remained to be learned about differences in course between alcohol abuse and dependence. Below, we present the results of further research on this topic: a study of a community sample of men and women using fully-operationalized DSM-IV diagnostic criteria, comparing the short-term course of alcohol abuse and dependence. 


\section{METHODS}

\section{Sample}

Subjects for this study were household residents of a sociodemographically diverse area near New York City. The methods of the study have been presented in detail elsewhere (Hasin et al., 1996a; Hasin et al., 1996b). In brief, households were designated via random digit dialing. Randomly designated members of each household were screened for eligibility for the study, with oversampling for women. Eligibility (inclusion) criteria included drinking five or more drinks at least once in the year prior to screening, being within the ages of 18 and 65, and speaking English well enough to participate in the interview. Subjects were screened by telephone for eligibility through a very brief set of questions on general health behaviors such as exercise, smoking, weight and drinking. Eligibility status and screening were conducted on $81 \%$ of the designated households (possibly missing households with less educated members; see below). Eligible subjects were asked to participate in a more extensive in-person interview that provided the baseline diagnostic data for this study. Of those screened and eligible to participate, $92 \%$ participated. Of these, $90 \%$, or 876, participated in a second interview approximately a year later (mean time between interviews, 13.6 months) that provided the follow-up diagnostic data. There were no statistically significant differences between those followed up and those not followed in terms of age, gender, race, and average ethanol consumption at baseline.

\section{Subjects}

About $46 \%$ of the subjects were female. About $24 \%$ were below age 25 , another $21 \%$ between 25 and 29, 17\% between 30 and 34, 13\% between 35 and 39, and the rest (25\%) 40 and above. Only $6 \%$ had not completed high school, about $53 \%$ had a high school diploma or GED, and $41 \%$ had a college degree, limiting generalizeability to illiterate or very poorly educated individuals. Minority residents constituted about $20 \%$ of the sample, the approximate proportion of the minority population of the area in which the study took place. About two-thirds of the minority subjects were African-American, with the remainder Hispanic and other ethnic groups. Most of the subjects (two-thirds) worked full time at baseline. About half (53\%) were never married, 37\% were married, and most of the rest were divorced. The young age and unmarried status of many of the respondents was expected, given that household residents were screened for heavier than average drinking without further oversampling for older age and marital status.

\section{Measures}

The Alcohol Use Disorders and Associated Disabilities Interview Schedule (AUDADIS; Grant et al., 1995) was used to assess the symptoms and criteria of alcohol use disorders. The AUDADIS is a fully-structured interview that was designed for administration by lay interviewers in a large NIAAA-sponsored U.S. national survey conducted in 1992 (Grant et al., 1994). In the AUDADIS, the symptoms and criteria of alcohol use disorders are covered in detail for the prior 12 months (current) and for the past. AUDADIS diagnoses of alcohol dependence require that symptoms cluster together in time, representing a syndrome of symptoms. While the AUDADIS was developed prior to publication of DSM- 
IV, advisory contact with the DSM-IV substance disorder work group provided information on the necessary diagnostic criteria for DSM-IV diagnoses, and these were incorporated in the interview. Computer algorithms developed for the AUDADIS operationalize the diagnostic criteria. For the follow-up interview (developed for the present study), the timeframe of AUDADIS was adjusted so that coverage included drinking and the occurrence of the criteria for alcohol use disorders between the baseline interview and the follow-up.

In a separate study of household residents (Grant et al., 1995), good test-retest reliability coefficients were obtained for alcohol disorder diagnoses as measured by the AUDADIS. To demonstrate reliability in the present sample, we conducted an additional test-retest AUDADIS reliability study with 50 subjects who participated in the main study. These subjects were a consecutive series of subjects who received their retest interview approximately a week after they participated in their baseline interview for the study. Reliability for current DSM-IV alcohol dependence (indicated by kappa) was .81 (s.e. .09), and reliability for current DSM-IV alcohol abuse was .48 (s.e. .32).

In DSM-IV, a diagnosis of alcohol dependence is assigned if three or more dependence symptoms clustered within a one-year period. A diagnosis of "partial remission" is assigned if individuals met full criteria for dependence at any time in the past, and currently have either one or two symptoms of dependence or meet criteria for abuse. According to DSM-IV, individuals cannot be given the diagnosis of abuse if they ever met criteria for dependence, even if a lengthy period of total remission separates the earlier period of dependence from the subsequent period of abuse. Thus, in this study, individuals diagnosed with abuse at baseline never met criteria for dependence in the past. Our baseline diagnoses of dependence included those currently in partial remission: one or two symptoms in the year prior to the interview and full criteria met within a one-year period at some point in the past. Our follow-up diagnoses generally employed a similar logic: dependence was diagnosed if three or more dependence symptoms were present in the interval between baseline and follow-up interview, or if criteria for dependence had been met previously and one or two dependence criteria (or abuse) were reported between baseline and follow-up. We did make one exception to the DSM-IV rules in creating the follow-up diagnoses. Cases who had no diagnosis at baseline and who met criteria for abuse at follow-up were given the diagnosis of abuse at follow-up, regardless of whether they had met criteria for dependence at some point in the past. We did this to make our present data directly comparable to the data from our earlier study (Hasin et al., 1990; Hasin et al., letter).

\section{Analyses}

Differences in outcome between diagnostic groups as represented by baseline diagnoses were tested with chi-square tests. As noted, follow-up diagnostic categories were created to replicate the earlier study as closely as possible. These included (1) no diagnosis, (2) "abuse": meeting cross-sectional criteria for abuse but no dependence symptoms, and (3) dependence: full dependence criteria, or partial remission consisting of one or two dependence symptoms among subjects who previously met full dependence criteria. Multinomial linear logistic regression analyses were conducted to test for group differences at follow-up controlling for potential confounders. In multinomial analyses, one outcome category is selected as the "reference" category, although this choice does not 
alter the mathematical results. We used the "no diagnosis" outcome category as the reference in the multinomial analyses, estimating the significance of diagnostic and control predictors of abuse or dependence at follow-up compared to no diagnosis. Control variables included age, gender, race, and length of time between interviews, since this varied between subjects.

\section{RESULTS}

Table I shows the baseline and follow-up status of the subjects. As shown, subjects who initially met criteria for alcohol abuse were unlikely to develop alcohol dependence between their baseline and follow-up interview. A substantial majority reported no symptoms of alcohol abuse or dependence between the baseline and follow-up interview. About a third of those meeting criteria for abuse at baseline remained in this category at followup. The course of those with abuse did differ significantly from those who had no diagnosis at baseline $\left(x^{2}=73.4, d f=2, p<.0001\right)$, since very few of the group with no diagnosis at baseline developed alcohol abuse during the follow-up.

In contrast, the course of alcohol dependence in this community sample was quite different. Table 1 shows that two-thirds of those meeting criteria for dependence at baseline remained in this category at follow-up. The artificial "abuse" category was created to allow the most complete replication of the earlier study, but very few subjects fell into this category at follow-up. According to DSM-IV diagnostic criteria for dependence, these subjects would also be considered dependent at follow-up, since abuse constitutes partial remission from dependence once the dependence diagnosis has been made. Finally, 29\% of those meeting criteria for dependence at baseline were considered in full remission at follow-up. The difference in outcome between the abuse and dependence groups was statistically significant $\left(\mathrm{X}^{2}=94.6, d f=2, p<.0001\right)$. When the entire $3 \times 3$ table is considered as a whole, the follow-up results remain significantly different for all groups $\left(\mathrm{X}^{2}=429.9\right.$, $d f=4, p<.0001$ ).

Table 2 shows the results of the multinomial logistic regression analyses in which baseline diagnoses were used as predictors of follow-up status controlling for age, gender, race and length of time between interviews. When considering the significance levels of the predictors as well as the betas (which give the direction and some indication of the size of the effect), alcohol abuse at baseline was a strong predictor of alcohol abuse at follow-up, although race (being white) and gender (being female) also exerted significant effects on

TABLE 1

Follow-up Status of Community Heavy Drinkers $(N=876)$

\begin{tabular}{lccc}
\hline & No Diagnosis/ \\
Remitted & Follow-Up & \\
Baseline & .88 & .04 & Dependence \\
\hline No Diagnosis (570) & .61 & .33 & .07 \\
Abuse (67) & .29 & .04 & .06 \\
Dependence (239) & Abuse & .67 \\
\hline
\end{tabular}

*For subjects dependent at baseline, this category includes those who met cross-sectional criteria for only abuse at follow-up. 
TABLE 2

Follow-up Diagnoses Predicted by Baseline Diagnoses, Controlling for Potential Confounders: Multinomial Logistic Regression Analyses $(\mathrm{N}=876)$

\begin{tabular}{|c|c|c|c|c|}
\hline \multirow[b]{3}{*}{ Baseline Predictors } & \multicolumn{4}{|c|}{ Follow-Up Status } \\
\hline & \multicolumn{2}{|c|}{$\begin{array}{l}\text { Abuse Compared } \\
\text { to No Diagnosis }\end{array}$} & \multicolumn{2}{|c|}{$\begin{array}{c}\text { Dependence Compared } \\
\text { to No Diagnosis }\end{array}$} \\
\hline & $b($ s.e. $)$ & $P$ & $b($ s.e. $)$ & $P$ \\
\hline Alcohol Abuse & $2.212(.36)$ & .000 & $.087(.55)$ & .875 \\
\hline Alcohol Dependence & --- & & $3.313(.21)$ & .000 \\
\hline Race (white/non white) & $1.346(.64)$ & .035 & $.196(.27)$ & .466 \\
\hline Age & $-.020(.02)$ & .225 & $-.011(.01)$ & .270 \\
\hline Sex & $-1.984(.50)$ & .000 & $-.142(.21)$ & .501 \\
\hline Length of Follow-Up & $.001(.00)$ & .583 & $.001(.00)$ & 400 \\
\hline
\end{tabular}

the likelihood of abuse at follow-up. When considering dependence, Table 2 shows that alcohol dependence at baseline was a strong, significant predictor of alcohol dependence at follow-up. In contrast to alcohol abuse, none of the control variables had a significant relationship with alcohol dependence when baseline dependence was included in the model. Note that these analyses were conducted without the artificial "abuse" category as a possible outcome for dependence, in accordance with the actual DSM-IV criteria.

To gain a better understanding of the group that had been diagnosed with alcohol abuse at baseline, we examined the diagnostic criteria that gave subjects the abuse diagnosis at baseline and conducted some post hoc analyses of follow-up status by the abuse symptoms manifested at baseline. Most subjects met criteria for abuse by evidencing only one abuse symptom. The most common abuse symptom by far was hazardous driving, evidenced by $78 \%$ of the subjects who met criteria for abuse. Since the AUDADIS ascertains the approximate frequency of occurrence of each dependence and abuse symptom, we further examined the frequency of this symptom, in the 12 months prior to the baseline interview and during the follow-up period. The mean number of occurrences was quite high (13 times) during the year prior to the baseline interview, due in large part to a few individuals who said they did this every day or nearly every day. The median and mode were 4.5 times per year. Among those who continued to meet abuse criteria by driving drunk during the follow-up period, the mean was nine times and the median and mode were again 4.5 times.

No abuse symptom was significantly predictive of continued abuse at follow-up. One abuse symptom, failing to meet role obligations due to recurrent drinking, predicted the few abuse cases that developed into dependence at follow-up $\left(\mathrm{X}^{2}=8.67, d f=1, p=.003\right)$. Due to the number of tests run and the very small number of cases that contributed to this test result, the finding would probably not be worth noting except for the fact that this criterion had been a dependence symptom in DSM-III-R but was changed into an abuse symptom in DSM-IV.

\section{DISCUSSION}

This study provides evidence on a number of points concerning the short-term course of alcohol abuse and dependence in an untreated sample. First, the course of abuse and depen- 
dence differ markedly and significantly. Those with alcohol dependence at baseline were quite likely to remain in this category at follow-up, with very few reverting to the artificially-constructed "abuse" status. According to the Guze validation paradigm, this lends support to the validity of the alcohol dependence diagnosis. In contrast, subjects with diagnoses of alcohol abuse at baseline were very unlikely to become alcohol dependent by follow-up. In fact, they were no more likely to do so than those with no alcohol diagnosis at all at baseline. Instead, for subjects with a diagnosis of abuse at baseline, the most common outcome at follow-up was no alcohol diagnosis at all, although about a third of the abusers remained in this category at their follow-up interview.

Our post hoc analyses showed that despite the expansion of DSM-IV criteria for the abuse diagnosis, most subjects received this diagnosis with only one criterion: repeated drunk driving. This behavior can clearly lead to serious injury for the subjects or others. However, the transience (high remission rate) of this category and the youth of the sample suggests that greater maturity may alleviate this behavior as it does other risky adolescent behaviors that sometimes persist into late adolescence and early adulthood.

While this article has focussed on questions concerning abuse, one of the principal aims of the longitudinal study was to determine whether the alcohol dependence diagnosis appeared valid when applied outside a treated sample. This report lends further weight to evidence from cross-sectional research (Hasin et al., 1996b; Hasin et al., 1997) that the dependence diagnosis is indeed valid in this group, given the differences in course between those with dependence and those with either alcohol abuse or no alcohol diagnosis.

A much higher proportion of respondents received a diagnosis of alcohol dependence at baseline in this study than in the earlier study (Hasin et al., 1990). The prevalence of alcohol dependence was almost certainly increased because of the screening procedure we used to identify subjects for the study. All current drinkers were included in the earlier study, with no screening for heavy drinking. The screening for the current study was implemented to produce a gender-balanced community sample with an increased level of alcohol use disorders while keeping costs down. The screening criteria were developed through analyses (Hasin, unpublished) of national data (Hilton, 1987) to reduce the number of subjects who were uninformative concerning current alcohol problems and disorders due to low levels of drinking. In addition,full coverage of the diagnostic criteria for DSM-IV alcohol dependence, which was not possible in the earlier study, may have increases the prevalence of cases somewhat.

Table 1 shows that $11 \%$ of the sample who had no alcohol diagnosis at baseline received a diagnosis of either abuse or dependence at follow-up. This would be a high onset rate in a sample that had not been screened for elevated, at-risk drinking. However, given the screening method, the onset rate is not so surprising. In addition, some of the cases who had no diagnosis of an alcohol use disorder at baseline had received a diagnosis of past disorder that had occurred and fully remitted by the time of the baseline interview. About half of the "new" onsets between baseline and follow-up came from this group.

Much past alcohol research has been criticized on the grounds that women are either excluded or included in numbers too small to analyze. Producing a community sample of heavy drinkers that is nearly balanced for gender requires considerable extra effort, since many more women than men must be screened to arrive at the final sample. The multinomial logistic regression analyses indicated that among those with an alcohol abuse 
diagnosis at baseline, men were more likely than women to remain in this category at follow-up, a finding borne out by the numbers: $41 \%$ of the male alcohol abusers continued in the abuse category, while only $14 \%$ of the female alcohol abusers did so. A similar pattern was found for whites compared to non-whites, although the group differences were not as marked. Despite these gender and race/ethnicity effects when included as control variables, demographic characteristics did not override the strong effects of the diagnostic categories themselves as predictors of outcome, and did not extend to dependence diagnoses. The issue of gender effects on outcomes of alcohol use disorders is complex and deserves further study. However, it is outside the scope of the present paper and will be considered further elsewhere.

This study employed a shorter length of follow-up than the earlier study. While the more complete and accurate representation of the diagnostic criteria for abuse and dependence may have led to a greater tendency to remain "true" within diagnostic categories during follow-up, the stability within categories may also have been caused by the shorter period in which to shift. An additional follow-up of this sample would enable us to address this question by combining data on events remembered relatively well due to a short period of follow-up with information about a more extended period of time. We hope to be able to conduct such a follow-up to provide longer-term information about the course of disorders in this sample.

Due to the nature of the sample design, we cannot generalize the results to special samples such as the elderly, psychiatric patients, or particular ethnic groups not represented in the area of the study. Conducting this type of research with these population subgroups would provide additional information on the applicability of the findings across a wide variety of particular groups. In addition, the generalizeability of this work to substances other than alcohol remains an open question. While outside the scope of the present study, such research would also add to our understanding of the validity of these diagnostic categories.

ACKNOWLEDGMENTS: This research was supported by grants R01 08159, K02 AA00161, and the New York State Department of Mental Hygiene.

\section{REFERENCES}

Cahalan, D., \& Room, R. (1974). Problem drinking among American men. New Brunswick, NJ: Rutgers Center of Alcohol Studies.

Edwards, G., \& Gross, M. (1976). Alcohol dependence: provisional description of a clinical syndrome. British Medical Journal, 1, 1058-1061.

Fillmore, K. (1987). Prevalence, incidence and chronicity of drinking patterns and problems among men as a function of age: A longitudinal and cohort analysis. British Journal ofAddictions, 82, 77-83.

Grant, B., Harford, T., Dawson, D., Chou, P., \& Pickering, R. (In press). The AUDADIS: Reliability of alcohol and drug modules in a general population sample. Drug and Alcohol Dependence.

Grant, B., Harford, T., Dawson, D., Chou, P., Dufour, M., \& Pickering, R. (1994). Prevalence of DSM-IV alcohol abuse and dependence: United States, 1992. Alcohol Health Research World, 18, 243-248.

Hasin, D., Grant, B., \& Endicott, J. (1990). Natural history of alcohol abuse: implications for definitions of alcohol use disorders. American Journal of Psychiatry, 147, 1537-1540. 
Hasin, D., Grant, B., Endicott, J. (1991 a). Letter. A merican Journal of Psychiatry, 148, 1096.

Hasin, D., Grant, B., Endicott, J. (1991b). Letter. A merican Journal of Psychiatry, 148, 1410-1411.

Hasin, D., Li, Q., McCloud, S., \& Endicott, J. (1996a). Agreement between DSM-III, DSM-III-R, DSM-IV and ICD-10 alcohol diagnoses in community-sample heavy drinkers. Addiction, 91, 1517-1527.

Hasin, D., McCloud, S., Li, Q., Endicott, J. (1996b). Cross-system agreement among demographic subgroups: DSM-III, DSM-III-R, DSM-IV and ICD-10 diagnosis of alcohol use disorders. Drug and Alcohol Dependence, 41, 127-135.

Hasin, D., Van Rossem, R., McCloud, S., \& Endicott, J. (1997). Alcohol dependence and abuse diagnoses: Cross-sectional validity in community sample heavy drinkers. Alcoholism: Clinical and Experimental Research, 21, 213-219.

Hilton, M. (1987). Drinking patterns and drinking problems in 1984: Results from a general population survey. Alcoholism: Clinical Experimental Research, 11, 167-175.

Robins, E., \& Guze, S. (1970). Establishment of diagnostic validity in psychiatric illness: Its application to schizophrenia. American Journal of Psychiatry, 126, 983-987.

Rounsaville, B., Spitzer, R., \& Williams, J. (1986). Proposed changes in DSM-III substance use disorders: description and rationale. A merican Journal of Psychiatry, 143, 463-468.

World Health Organization. (1977). Report of a WHO group of investigators on criteria for identifying and classifying disabilities related to alcohol consumption. In G. Edwards, N. Gross, M. Keller, J. Moser, \& R. Room (Eds.). Alcohol-related disabilities (pp. 6-22). Geneva: Authors.

World Health Organization. (1981). Nomenclature and classification of drug- and alcohol-related problems: WHO memorandum. Bulletin of the World Health Organization, 99, 225-242. 